\section{Preparations for a Civil Disturbance by a Civilian Pediatric Transport Team}

${ }^{*} D$. van Stralen, ${ }^{1} M D, R$. Vannix, MSN, RN, ${ }^{1}$

T. Young, MSN, RN, ${ }^{1} T$. Zirkle, MD, Deputy Chief, ${ }^{2}$ R. Perkins, $M D,{ }^{1}$ B. Boswell, Sergeant, ${ }^{3}$ M. McKee, Captain, EMT-P, ${ }^{4}$ J. Martin, Captain, EMT-P, ${ }^{5}$ D. Camp, EMT-P

1. Center for Pediatric Prehospital Care, Loma Linda University, Loma Linda, California

2. San Bernardino Co. Sheriff's Department, California

3. Redlands Fire Department, California

4. Los Angeles City Fire Department, California

5. Loma Linda Fire Department, California

Purpose: Civil disturbances occur in the urban environment with threats of sniper fire, guerrilla warfare, and riot. Issues in preparing a civilian university hospitalbased pediatric and neonatal transport team for an expected civil disturbance were identified.

Methods: Prospective collection of incidents, reactions, impediments in preparing this system for a civil disturbance. Participants included law enforcement/emergency medical services (LE/EMS) planners, pediatric transport personnel, and hospital administrators.

Results: 1)Ambulance diversion due to ED saturation would prolong paramedic field time and expose them to life-threatening situations; 2) LE/EMS interactions allowed notification of threat to the transport team, integration into emergency operations, and communication with referral EDs; 3) adaptability was encouraged by selective suspension of usual protocols, policies, or procedures; 4) arranged recall of the intensive care unit (ICU) transport staff; 5) psychological and personalsafety counseling of the transport team; and 6) hospital administrative preparation included coordinating administrative structure with LE/EMS, team safety (job security, lodging, meals), and legal issues (labor, insurance, consent).

Conclusion: The ICU is not automatically incorporated into LE/EMS evacuation plans. The transport service must address several issues: 1) coordination with LE/EMS is mandatory; 2) transport services must identify whether they can adapt to LE/EMS and provide indicated medical care in the changing constraints of a civil disturbance; 3) the threats to patient and team for each transport must be identified; and 4) the unique advantages, disadvantages, and hazards of helicopter use during a civil disturbance must be recognized.

\section{Assessment of the Usefulness of Peak Expiratory Flow Rate to Differentiate Out- of-Hospital CHF and COPD Patients}

\author{
*James M. Keller, MD, ${ }^{l}$ Theodore R. Delbridge, \\ $M D,^{2}$ Michael P. Sullivan, MD, ${ }^{1}$ Alan $K$. \\ Hodgdon, $M D,^{3}$ Thomas E. Auble, $P h D^{2}$ \\ 1. University of Pittsburgh, Affiliated Residency in Emergency \\ Medicine, Pittsburgh, Pennsylvania \\ 2. University of Pittsburgh Division of Emergency Medicine, \\ Pittsburgh, Pennsylvania \\ 3 Mercy Hospital of Pittsburgh, Department of Emergency \\ Medicine, Pittsburgh, Pennsylvania
}

Purpose: Patients with shortness of breath often present diagnostic dilemmas for EMS personnel. A previous study found that peak expiratory flow rates (PEFR) in emergency department (ED) congestive heart failure (CHF) and chronic lung disease (COPD) patients differed by greater than $100 \mathrm{~L} / \mathrm{min}(224 \pm 82$ and $108 \pm 49$ $\mathrm{L} / \mathrm{min}$ respectively). The current study assessed the usefulness of PEFR measurements to differentiate out-ofhospital CHF and COPD patients.

Methods: The study was a prospective, case series in an urban, municipal EMS system during three weeks in August 1993. Participants were out-of-hospital patients, age $>40$ years, with a chief complaint of shortness of breath. Patients excluded were those that could not cooperate, required immediate therapy, or did not give informed consent. In addition to history physical examination, paramedics recorded the degree of respiratory distress (mild, moderate, severe, or critical), greatest of three PEFR measurements, and patient effort (poor, fair, or good). Results were not used to guide therapy, the final diagnosis was that determined by the receiving ED physician.

Results: Forty-eight patients were enrolled; nine were excluded for poor effort and nine for final diagnosis other than CHF or COPD. Gender distribution was similar in the CHF and COPD groups (Fisher's Exact Test, $p=0.12$ ), but age was greater in the COPD group ( $76 \pm 6$ vs. $66 \pm 11$ years, $t$-test: $p=.015)$. Independent $t$-test revealed no significant differences $(p=-0.48)$ between CHF ( $\mathrm{n}=14)$ and COPD ( $=16)$ PEFR $(150 \pm 92$ and $127 \pm 77 \mathrm{~L} / \mathrm{min}$, respectively). Analysis of only moderate $/$ severe distress patients $(\mathbf{n}=26)$ was similar $(p=$ 0.94 ). The study had $80 \%$ power to detect a $100 \mathrm{~L} / \mathrm{min}$ difference in PEFR between CHF and COPD groups (alpha $=0.05)$.

Conclusions: This study does not support the use of PEFR to differentiate out-of-hospital CHF and COPD patients. Further study might evaluate the effects of the age difference found between the two groups. 